\title{
GENERALIZED $(\mathscr{F}, b, \phi, \rho, \theta)$-UNIVEX $n$-SET FUNCTIONS AND PARAMETRIC DUALITY MODELS IN MINMAX FRACTIONAL SUBSET PROGRAMMING
}

\author{
G. J. ZALMAI
}

Received 28 December 2003 and in revised form 3 October 2004

We construct three parametric duality models and establish a fairly large number of duality results under a variety of generalized $(\mathscr{F}, b, \phi, \rho, \theta)$-univexity assumptions for a discrete minmax fractional subset programming problem.

\section{Introduction}

In this paper, we will formulate three parametric duality models and prove a variety of duality results under various generalized $(\mathscr{F}, b, \phi, \rho, \theta)$-univexity hypotheses for the following discrete minmax fractional subset programming problem:

$$
\text { Minimize } \max _{1 \leq i \leq p} \frac{F_{i}(S)}{G_{i}(S)} \text { subject to } H_{j}(S) \leq 0, j \in \underline{q}, S \in \mathbb{A}^{n},
$$

where $\mathbb{A}^{n}$ is the $n$-fold product of the $\sigma$-algebra $\mathbb{A}$ of subsets of a given set $X, F_{i}, G_{i}$, $i \in \underline{p} \equiv\{1,2, \ldots, p\}$, and $H_{j}, j \in \underline{q}$, are real-valued functions defined on $\mathbb{A}^{n}$, and for each $i \in \bar{p}, G_{i}(S)>0$ for all $S \in \mathbb{A}^{n}$ such that $H_{j}(S) \leq 0, j \in q$.

This problem was considered previously in the companion paper [7] where a survey of currently available optimality and duality results for minmax fractional susbset programming problems was presented, a fairly comprehensive list of references dealing with different aspects of these problems was provided, several new classes of generalized convex $n$-set functions were defined, and numerous sets of global parametric sufficient optimality conditions under various generalized $(\mathscr{F}, b, \phi, \rho, \theta)$-univexity assumptions were established. In the present study, we construct some dual problems for $(\mathrm{P})$ and prove appropriate duality theorems utilizing most of the new classes of generalized $n$-set convex functions that were introduced in [7].

The rest of this paper is organized as follows. In Section 2, we recall the definitions of differentiability and certain types of generalized convexity for $n$-set functions which will be used frequently throughout the sequel. We begin our discussion of duality for (P) in Section 3 where we formulate a simple dual problem and prove weak, strong, and strict converse duality theorems. In Section 4, we consider another dual problem 
with a relatively flexible constraint structure that allows for a greater variety of generalized $(\mathscr{F}, b, \phi, \rho, \theta)$-univexity hypotheses under which duality can be established. Finally, in Section 5, we state and discuss a general duality model which is, in fact, a family of dual problems for $(\mathrm{P})$ whose members can readily be identified by appropriate choices of certain sets and functions. The nonparametric counterparts of the duality results established in this paper are investigated in [5].

Evidently, all these duality results are also applicable, when appropriately specialized, to the following three classes of problems with discrete max, fractional, and conventional objective functions, which are particular cases of $(\mathrm{P})$ :

$$
\begin{gathered}
\underset{S \in \mathbb{F}}{\operatorname{Minimize}} \max _{1 \leq i \leq p} F_{i}(S), \\
\underset{S \in \mathbb{F}}{\operatorname{Minimize}} \frac{F_{1}(S)}{G_{1}(S)}, \\
\underset{S \in \mathbb{F}}{\operatorname{Minimize}} F_{1}(S),
\end{gathered}
$$

where $\mathbb{F}$ (assumed to be nonempty) is the feasible set of $(\mathrm{P})$, that is,

$$
\mathbb{F}=\left\{S \in \mathbb{A}^{n}: H_{j}(S) \leq 0, j \in \underline{q}\right\}
$$

Since, in most cases, the duality results established for $(\mathrm{P})$ can easily be modified and restated for each one of the above problems, we will not explicitly state these results.

\section{Preliminaries}

In this section, we gather, for convenience of reference, a few basic definitions and auxiliary results which will be used often throughout the paper.

Let $(X, \mathbb{A}, \mu)$ be a finite atomless measure space with $L_{1}(X, \mathbb{A}, \mu)$ separable, and let $d$ be the pseudometric on $\mathbb{A}^{n}$ defined by

$$
d(R, S)=\left[\sum_{i=1}^{n} \mu^{2}\left(R_{i} \triangle S_{i}\right)\right]^{1 / 2}, \quad R=\left(R_{1}, \ldots, R_{n}\right), S=\left(S_{1}, \ldots, S_{n}\right) \in \mathbb{A}^{n}
$$

where $\triangle$ denotes symmetric difference; thus $\left(\mathbb{A}^{n}, d\right)$ is a pseudometric space. For $h \in$ $L_{1}(X, \mathbb{A}, \mu)$ and $T \in \mathbb{A}$ with characteristic function $\chi_{T} \in L_{\infty}(X, \mathbb{A}, \mu)$, the integral $\int_{T} h d \mu$ will be denoted by $\left\langle h, \chi_{T}\right\rangle$.

We next define the notion of differentiability for $n$-set functions. It was originally introduced by Morris [3] for a set function, and subsequently extended by Corley [1] for $n$-set functions.

Definition 2.1. A function $F: \mathbb{A} \rightarrow \mathbb{R}$ is said to be differentiable at $S^{*}$ if there exists $D F\left(S^{*}\right)$ $\in L_{1}(X, \mathbb{A}, \mu)$, called the derivative of $F$ at $S^{*}$, such that for each $S \in \mathbb{A}$,

$$
F(S)=F\left(S^{*}\right)+\left\langle D F\left(S^{*}\right), \chi_{S}-\chi_{S^{*}}\right\rangle+V_{F}\left(S, S^{*}\right),
$$

where $V_{F}\left(S, S^{*}\right)$ is $o\left(d\left(S, S^{*}\right)\right)$, that is, $\lim _{d\left(S, S^{*}\right) \rightarrow 0} V_{F}\left(S, S^{*}\right) / d\left(S, S^{*}\right)=0$. 
Definition 2.2. A function $G: \mathbb{A}^{n} \rightarrow \mathbb{R}$ is said to have a partial derivative at $S^{*}=\left(S_{1}^{*}, \ldots, S_{n}^{*}\right)$ $\in \mathbb{A}^{n}$ with respect to its $i$ th argument if the function $F\left(S_{i}\right)=G\left(S_{1}^{*}, \ldots, S_{i-1}^{*}, S_{i}, S_{i+1}^{*}, \ldots, S_{n}^{*}\right)$ has derivative $\operatorname{DF}\left(S_{i}^{*}\right), i \in \underline{n}$; in that case, the $i$ th partial derivative of $G$ at $S^{*}$ is defined to be $D_{i} G\left(S^{*}\right)=D F\left(S_{i}^{*}\right), i \in \underline{n}$.

Definition 2.3. A function $G: \mathbb{A}^{n} \rightarrow \mathbb{R}$ is said to be differentiable at $S^{*}$ if all the partial derivatives $D_{i} G\left(S^{*}\right), i \in \underline{n}$, exist and

$$
G(S)=G\left(S^{*}\right)+\sum_{i=1}^{n}\left\langle D_{i} G\left(S^{*}\right), \chi_{S_{i}}-\chi_{S_{i}^{*}}\right\rangle+W_{G}\left(S, S^{*}\right),
$$

where $W_{G}\left(S, S^{*}\right)$ is $o\left(d\left(S, S^{*}\right)\right)$ for all $S \in \mathbb{A}^{n}$.

We next recall the definitions of the generalized $(\mathscr{F}, b, \phi, \rho, \theta)$-univex $n$-set functions which will be used in the statements of our duality theorems. For more information about these and a number of other related classes of $n$-set functions, the reader is referred to [7]. We begin by defining a sublinear function which is an integral part of all the subsequent definitions.

Definition 2.4. A function $\mathscr{F}: \mathbb{R}^{n} \rightarrow \mathbb{R}$ is said to be sublinear (superlinear) if $\mathscr{F}(x+y) \leq$ $(\geq) \mathscr{F}(x)+\mathscr{F}(y)$ for all $x, y \in \mathbb{R}^{n}$, and $\mathscr{F}(a x)=a \mathscr{F}(x)$ for all $x \in \mathbb{R}^{n}$ and $a \in \mathbb{R}_{+} \equiv[0, \infty)$.

Let $S, S^{*} \in \mathbb{A}^{n}$, and assume that the function $F: \mathbb{A}^{n} \rightarrow \mathbb{R}$ is differentiable at $S^{*}$.

Definition 2.5. The function $F$ is said to be (strictly) $(\mathscr{F}, b, \phi, \rho, \theta)$-univex at $S^{*}$ if there exist a sublinear function $\mathscr{F}_{(}\left(S, S^{*} ; \cdot\right): L_{1}^{n}(X, \mathbb{A}, \mu) \rightarrow \mathbb{R}$, a function $b: \mathbb{A}^{n} \times \mathbb{A}^{n} \rightarrow \mathbb{R}$ with positive values, a function $\theta: \mathbb{A}^{n} \times \mathbb{A}^{n} \rightarrow \mathbb{A}^{n} \times \mathbb{A}^{n}$ such that $S \neq S^{*} \Rightarrow \theta\left(S, S^{*}\right) \neq(0,0)$, a function $\phi: \mathbb{R} \rightarrow \mathbb{R}$, and a real number $\rho$ such that for each $S \in \mathbb{A}^{n}$,

$$
\phi\left(F(S)-F\left(S^{*}\right)\right)(>) \geq \mathscr{F}\left(S, S^{*} ; b\left(S, S^{*}\right) D F\left(S^{*}\right)\right)+\rho d^{2}\left(\theta\left(S, S^{*}\right)\right)
$$

Definition 2.6. The function $F$ is said to be (strictly) $(\mathscr{F}, b, \phi, \rho, \theta)$-pseudounivex at $S^{*}$ if there exist a sublinear function $\mathscr{F}\left(S, S^{*} ; \cdot\right): L_{1}^{n}(X, \mathbb{A}, \mu) \rightarrow \mathbb{R}$, a function $b: \mathbb{A}^{n} \times \mathbb{A}^{n} \rightarrow$ $\mathbb{R}$ with positive values, a function $\theta: \mathbb{A}^{n} \times \mathbb{A}^{n} \rightarrow \mathbb{A}^{n} \times \mathbb{A}^{n}$ such that $S \neq S^{*} \Rightarrow \theta\left(S, S^{*}\right) \neq$ $(0,0)$, a function $\phi: \mathbb{R} \rightarrow \mathbb{R}$, and a real number $\rho$ such that for each $S \in \mathbb{A}^{n}\left(S \neq S^{*}\right)$,

$$
\mathscr{F}\left(S, S^{*} ; b\left(S, S^{*}\right) D F\left(S^{*}\right)\right) \geq-\rho d^{2}\left(\theta\left(S, S^{*}\right)\right) \Longrightarrow \phi\left(F(S)-F\left(S^{*}\right)\right)(>) \geq 0
$$

Definition 2.7. The function $F$ is said to be $(\mathscr{F}, b, \phi, \rho, \theta)$-quasiunivex at $S^{*}$ if there exist a sublinear function $\mathscr{F}\left(S, S^{*} ; \cdot\right): L_{1}^{n}(X, \mathbb{A}, \mu) \rightarrow \mathbb{R}$, a function $b: \mathbb{A}^{n} \times \mathbb{A}^{n} \rightarrow \mathbb{R}$ with positive values, a function $\theta: \mathbb{A}^{n} \times \mathbb{A}^{n} \rightarrow \mathbb{A}^{n} \times \mathbb{A}^{n}$ such that $S \neq S^{*} \Rightarrow \theta\left(S, S^{*}\right) \neq(0,0)$, a function $\phi: \mathbb{R} \rightarrow \mathbb{R}$, and a real number $\rho$ such that for each $S \in \mathbb{A}^{n}$,

$$
\phi\left(F(S)-F\left(S^{*}\right)\right) \leq 0 \Longrightarrow \mathscr{F}\left(S, S^{*} ; b\left(S, S^{*}\right) D F\left(S^{*}\right)\right) \leq-\rho d^{2}\left(\theta\left(S, S^{*}\right)\right) .
$$


Definition 2.8. The function $F$ is said to be prestrictly $(\mathscr{F}, b, \phi, \rho, \theta)$-quasiunivex at $S^{*}$ if there exist a sublinear function $\mathscr{F}\left(S, S^{*} ; \cdot\right): L_{1}^{n}(X, \mathbb{A}, \mu) \rightarrow \mathbb{R}$, a function $b: \mathbb{A}^{n} \times \mathbb{A}^{n} \rightarrow$ $\mathbb{R}$ with positive values, a function $\theta: \mathbb{A}^{n} \times \mathbb{A}^{n} \rightarrow \mathbb{A}^{n} \times \mathbb{A}^{n}$ such that $S \neq S^{*} \Rightarrow \theta\left(S, S^{*}\right) \neq$ $(0,0)$, a function $\phi: \mathbb{R} \rightarrow \mathbb{R}$, and a real number $\rho$ such that for each $S \in \mathbb{A}^{n}, S \neq S^{*}$,

$$
\phi\left(F(S)-F\left(S^{*}\right)\right)<0 \Longrightarrow \mathscr{F}\left(S, S^{*} ; b\left(S, S^{*}\right) D F\left(S^{*}\right)\right) \leq-\rho d^{2}\left(\theta\left(S, S^{*}\right)\right) .
$$

From the above definitions, it is clear that if $F$ is $(\mathscr{F}, b, \phi, \rho, \theta)$-univex at $S^{*}$, then it is both $(\mathscr{F}, b, \phi, \rho, \theta)$-pseudounivex and $(\mathscr{F}, b, \phi, \rho, \theta)$-quasiunivex at $S^{*}$, if $F$ is $(\mathscr{F}, b, \phi, \rho, \theta)$ quasiunivex at $S^{*}$, then it is prestrictly $(\mathscr{F}, b, \phi, \rho, \theta)$-quasiunivex at $S^{*}$, and if $F$ is strictly $(\mathscr{F}, b, \phi, \rho, \theta)$-pseudounivex at $S^{*}$, then it is $(\mathscr{F}, b, \phi, \rho, \theta)$-quasiunivex at $S^{*}$.

In the proofs of the duality theorems, sometimes it may be more convenient to use certain alternative but equivalent forms of the above definitions. These are obtained by considering the contrapositive statements. For example, $(\mathscr{F}, b, \phi, \rho, \theta)$-quasiunivexity can be defined in the following equivalent way: $F$ is said to be $(\mathscr{F}, b, \phi, \rho, \theta)$-quasiunivex at $S^{*}$ if for each $S \in \mathbb{A}^{n}$,

$$
\mathscr{F}\left(S, S^{*} ; b\left(S, S^{*}\right) D F\left(S^{*}\right)\right)>-\rho d^{2}\left(\theta\left(S, S^{*}\right)\right) \Longrightarrow \phi\left(F(S)-F\left(S^{*}\right)\right)>0 .
$$

Needless to say that the new classes of generalized convex $n$-set functions specified in Definitions 2.5-2.8 contain a variety of special cases; in particular, they subsume all the previously defined types of generalized $n$-set functions. This can easily be seen by appropriate choices of $\mathscr{F}, b, \phi, \rho$, and $\theta$.

We next recall a set of parametric necessary optimality conditions which will be needed for proving strong and strict converse duality theorems for $(\mathrm{P})$.

Theorem 2.9 [6]. Assume that $F_{i}, G_{i}, i \in \underline{p}$, and $H_{j}, j \in \underline{q}$, are differentiable at $S^{*} \in \mathbb{A}^{n}$, and there exists $\hat{S} \in \mathbb{A}^{n}$ such that

$$
H_{j}\left(S^{*}\right)+\sum_{k=1}^{n}\left\langle D_{k} H_{j}\left(S^{*}\right), \chi_{\hat{S}_{k}}-\chi_{S_{k}^{*}}\right\rangle<0, \quad j \in \underline{q} .
$$

If $S^{*}$ is an optimal solution of $(P)$, then there exist $u^{*} \in U, v^{*} \in \mathbb{R}_{+}^{q}$, and $\lambda^{*} \in \mathbb{R}$ such that

$$
\begin{gathered}
\left\langle\sum_{i=1}^{p} u_{i}^{*}\left[D_{k} F_{i}\left(S^{*}\right)-\lambda^{*} D_{k} G_{i}\left(S^{*}\right)\right]+\sum_{j=1}^{q} v_{j}^{*} D_{k} H_{j}\left(S^{*}\right), \chi_{S_{k}}-\chi_{S_{k}^{*}}\right\rangle \geq 0 \\
u_{i}^{*}\left[F_{i}\left(S^{*}\right)-\lambda^{*} G_{i}\left(S^{*}\right)\right]=0, \quad i \in \underline{p}, \\
v_{j}^{*} H_{j}\left(S^{*}\right)=0, \quad j \in \underline{\mathbb{q}},
\end{gathered}
$$

where $U=\left\{u \in \mathbb{R}_{+}^{p}: \sum_{i=1}^{p} u_{i}=1\right\}$ and $\mathbb{R}_{+}^{p}$ denotes the nonnegative orthant of $\mathbb{R}^{p}$.

For brevity, we will henceforth refer to an $S^{*} \in \mathbb{F}$ satisfying (2.9) as a regular feasible solution of $(\mathrm{P})$.

We will also need the following result which provides an alternative expression for the objective function of $(\mathrm{P})$. 
Lemma $2.10[6]$. For each $S \in \mathbb{A}^{n}$,

$$
\varphi(S) \equiv \max _{1 \leq i \leq p} \frac{F_{i}(S)}{G_{i}(S)}=\max _{u \in U} \frac{\sum_{i=1}^{p} u_{i} F_{i}(S)}{\sum_{i=1}^{p} u_{i} G_{i}(S)} .
$$

\section{Duality model I}

In this section, we discuss a duality model for $(\mathrm{P})$ with a somewhat restricted constraint structure that allows only certain types of generalized $(\mathscr{F}, b, \phi, \rho, \theta)$-univexity conditions for establishing duality. More general duality models will be presented in Sections 4 and 5 .

In the remainder of this paper, we assume that the functions $F_{i}, G_{i}, i \in \underline{p}$, and $H_{j}, j \in \underline{q}$, are differentiable on $\mathbb{A}^{n}$.

Let the functions $\mathscr{A}_{i}(\cdot, \lambda), \mathscr{A}(\cdot, u, \lambda)$, and $\mathscr{B}(\cdot, v): \mathbb{A}^{n} \rightarrow \mathbb{R}$ be defined, for fixed $\lambda, u$, and $v$, by

$$
\begin{gathered}
\mathscr{A}_{i}(S, \lambda)=F_{i}(S)-\lambda G_{i}(S), \quad i \in \underline{p}, \\
\mathscr{A}(S, u, \lambda)=\sum_{i=1}^{p} u_{i}\left[F_{i}(S)-\lambda G_{i}(S)\right], \\
\mathscr{B}(S, v)=\sum_{j=1}^{q} v_{j} H_{j}(S) .
\end{gathered}
$$

For given $u \in U$ and $v \in \mathbb{R}_{+}^{q}$, let $I_{+}(u)=\left\{i \in \underline{p}: u_{i}>0\right\}$ and $J_{+}(v)=\left\{j \in \underline{q}: v_{j}>0\right\}$.

Consider the following problem:

$$
\text { Maximize } \lambda
$$

subject to

$$
\begin{gathered}
\mathscr{F}\left(S, T ; \sum_{i=1}^{p} u_{i}\left[D F_{i}(T)-\lambda D G_{i}(T)\right]+\sum_{j=1}^{q} v_{j} D H_{j}(T)\right) \geq 0 \quad \forall S \in \mathbb{A}^{n}, \\
\sum_{i=1}^{p} u_{i}\left[F_{i}(T)-\lambda G_{i}(T)\right] \geq 0, \\
\sum_{j=1}^{q} v_{j} H_{j}(T) \geq 0, \\
T \in \mathbb{A}^{n}, \quad u \in U, \quad v \in \mathbb{R}_{+}^{q}, \quad \lambda \in \mathbb{R}_{+},
\end{gathered}
$$

where $\mathscr{F}(S, T ; \cdot): L_{1}^{n}(X, \mathbb{A}, \mu) \rightarrow \mathbb{R}$ is a sublinear function.

The following two theorems show that (DI) is a dual problem for $(\mathrm{P})$.

Theorem 3.1 (weak duality). Let $S$ and $(T, u, v, \lambda)$ be arbitrary feasible solutions of $(P)$ and (DI), respectively, and assume that any of the following three sets of hypotheses is satisfied:

(a) (i) for each $i \in \underline{p}, F_{i}$ is $\left(\mathscr{F}, b, \bar{\phi}, \bar{\rho}_{i}, \theta\right)$-univex at $T$, and $-G_{i}$ is $\left(\mathscr{F}, b, \bar{\phi}, \hat{\rho}_{i}, \theta\right)$ univex at $T, \bar{\phi}$ is superlinear, and $\bar{\phi}(a) \geq 0 \Rightarrow a \geq 0$; 
(ii) for each $j \in \underline{q}, H_{j}$ is $\left(\mathscr{F}, b, \tilde{\phi}_{j}, \tilde{\rho}_{j}, \theta\right)$-univex at $T, \tilde{\phi}_{j}$ is increasing, and $\tilde{\phi}_{j}(0)=$ 0 ;

(iii) $\rho^{*}+\sum_{j \in J_{+}} v_{j} \tilde{\rho}_{j} \geq 0$, where $\rho^{*}=\sum_{i=1}^{p} u_{i}\left(\bar{\rho}_{i}+\lambda \hat{\rho}_{i}\right)$;

(b) (i) for each $i \in \underline{p}, F_{i}$ is $\left(\mathscr{F}, b, \bar{\phi}, \bar{\rho}_{i}, \theta\right)$-univex at $T$, and $-G_{i}$ is $\left(\mathscr{F}, b, \bar{\phi}, \hat{\rho}_{i}, \theta\right)$ univex at $T, \bar{\phi}$ is superlinear, and $\bar{\phi}(a) \geq 0 \Rightarrow a \geq 0$;

(ii) $\mathscr{B}(\cdot, v)$ is $(\mathscr{F}, b, \tilde{\phi}, \tilde{\rho}, \theta)$-quasiunivex at $T, \tilde{\phi}$ is increasing, and $\tilde{\phi}(0)=0$;

(iii) $\rho^{*}+\tilde{\rho} \geq 0$;

(c) (i) $T \rightarrow \sum_{i=1}^{p} u_{i}\left[F_{i}(T)-\lambda G_{i}(T, u)\right]+\sum_{j=1}^{q} v_{j} H_{j}(T)$ is $(\mathscr{F}, b, \bar{\phi}, 0, \theta)$-pseudounivex at $T$, and $\bar{\phi}(a) \geq 0 \Rightarrow a \geq 0$.

Then $\varphi(S) \geq \lambda$.

Proof. (a) From (i) and (ii), it follows that

$$
\begin{aligned}
\bar{\phi}\left(F_{i}(S)-F_{i}(T)\right) \geq \mathscr{F}\left(S, T ; b(S, T) D F_{i}(T)\right)+\bar{\rho}_{i} d^{2}(\theta(S, T)), & i \in \underline{p}, \\
\bar{\phi}\left(-G_{i}(S)+G_{i}(T)\right) \geq \mathscr{F}\left(S, T ;-b(S, T) D G_{i}(T)\right)+\hat{\rho}_{i} d^{2}(\theta(S, T)), & i \in \underline{p}, \\
\tilde{\phi}\left(H_{j}(S)-H_{j}(T)\right) \geq \mathscr{F}\left(S, T ; b(S, T) D H_{j}(T)\right)+\tilde{\rho}_{j} d^{2}(\theta(S, T)), & j \in \underline{q} .
\end{aligned}
$$

Multiplying (3.6) by $u_{i}$ and (3.7) by $\lambda u_{i}, i \in p$, adding the resulting inequalities, and then using the superlinearity of $\bar{\phi}$ and sublinearity of $\mathscr{F}(S, T ; \cdot)$, we obtain

$$
\begin{gathered}
\bar{\phi}\left(\sum_{i=1}^{p} u_{i}\left[F_{i}(S)-\lambda G_{i}(S)\right]-\sum_{i=1}^{p} u_{i}\left[F_{i}(T)-\lambda G_{i}(T)\right]\right) \\
\geq \mathscr{F}\left(S, T ; b(S, T) \sum_{i=1}^{p} u_{i}\left[D F_{i}(T)-\lambda D G_{i}(T)\right]\right) \\
+\sum_{i=1}^{p} u_{i}\left(\bar{\rho}_{i}+\lambda \hat{\rho}_{i}\right) d^{2}(\theta(S, T)) .
\end{gathered}
$$

Likewise, from (3.8), we deduce that

$$
\tilde{\phi}\left(\sum_{j=1}^{q} v_{j}\left[H_{j}(S)-H_{j}(T)\right]\right) \geq \mathscr{F}\left(S, T ; b(S, T) \sum_{j=1}^{q} v_{j} D H_{j}(T)\right)+\sum_{j=1}^{q} v_{j} \tilde{\rho}_{j} d^{2}(\theta(S, T)) .
$$

Since $v \geq 0, S \in \mathbb{F}$, and (3.4) holds, it is clear that

$$
\sum_{j=1}^{q} v_{j}\left[H_{j}(S)-H_{j}(T)\right] \leq 0
$$

which implies, in view of the properties of $\tilde{\phi}$, that the left-hand side of (3.10) is less than or equal to zero, that is,

$$
0 \geq \mathscr{F}\left(S, T ; b(S, T) \sum_{j=1}^{q} v_{j} D H_{j}(T)\right)+\sum_{j=1}^{q} v_{j} \tilde{\rho}_{j} d^{2}(\theta(S, T)) .
$$


From the nonnegativity of $b(S, T)$, sublinearity of $\mathscr{F}(S, T ; \cdot)$, and $(3.2)$, it follows that

$$
\mathscr{F}\left(S, T ; b(S, T) \sum_{i=1}^{p} u_{i}\left[D F_{i}(T)-\lambda D G_{i}(T)\right]\right)+\mathscr{F}\left(S, T ; b(S, T) \sum_{j=1}^{q} v_{j} D H_{j}(T)\right) \geq 0 .
$$

Now adding (3.9) and (3.12), and then using (3.13) and (iii), we obtain

$$
\bar{\phi}\left(\sum_{i=1}^{p} u_{i}\left[F_{i}(S)-\lambda G_{i}(S)\right]-\sum_{i=1}^{p} u_{i}\left[F_{i}(T)-\lambda G_{i}(T)\right]\right) \geq 0 .
$$

But $\bar{\phi}(a) \geq 0 \Rightarrow a \geq 0$, and so (3.14) yields

$$
\sum_{i=1}^{p} u_{i}\left[F_{i}(S)-\lambda G_{i}(S)\right]-\sum_{i=1}^{p} u_{i}\left[F_{i}(T)-\lambda G_{i}(T)\right] \geq 0,
$$

which in view of (3.3) reduces to

$$
\sum_{i=1}^{p} u_{i}\left[F_{i}(S)-\lambda G_{i}(S)\right] \geq 0
$$

Making use of Lemma 2.10 and (3.16), we obtain the desired inequality as follows:

$$
\begin{aligned}
\varphi(S) & =\max _{1 \leq i \leq p} \frac{F_{i}(S)}{G_{i}(S)}=\max _{a \in U} \frac{\sum_{i=1}^{p} a_{i} F_{i}(S)}{\sum_{i=1}^{p} a_{i} G_{i}(S)} \quad \text { (by Lemma 2.10) } \\
& \geq \frac{\sum_{i=1}^{p} u_{i} F_{i}(S)}{\sum_{i=1}^{p} u_{i} G_{i}(S)} \geq \lambda \quad(\text { by }(3.16)) .
\end{aligned}
$$

(b) Since, for each $j \in \underline{q}, v_{j} H_{j}(S) \leq 0$, it follows from (3.4) that

$$
\sum_{j=1}^{q} v_{j} H_{j}(S) \leq 0 \leq \sum_{j=1}^{q} v_{j} H_{j}(T)
$$

and so using the properties of $\tilde{\phi}$, we obtain

$$
\tilde{\phi}\left(\sum_{j=1}^{q} v_{j} H_{j}(S)-\sum_{j=1}^{q} v_{j} H_{j}(T)\right) \leq 0,
$$

which in view of (ii) implies that

$$
\mathscr{F}\left(S, T ; b(S, T) \sum_{j=1}^{q} v_{j} D H_{j}(T)\right) \leq-\tilde{\rho} d^{2}(\theta(S, T)) .
$$

Now combining (3.9), (3.13), and (3.20), and using (iii), we obtain (3.16). Therefore, the rest of the proof is identical to that of part (a). 
(c) From the nonnegativity of $b(S, T)$, sublinearity of $\mathscr{F}(S, T ; \cdot),(\mathscr{F}, \bar{b}, \bar{\phi}, 0, \theta)$ pseudounivexity assumption, and (3.2), it follows that

$$
\bar{\phi}\left(\sum_{i=1}^{p} u_{i}\left[F_{i}(S)-\lambda G_{i}(S)\right]+\sum_{j=1}^{q} v_{j} H_{j}(S)-\left\{\sum_{i=1}^{p} u_{i}\left[F_{i}(T)-\lambda G_{i}(T)\right]+\sum_{j=1}^{q} v_{j} H_{j}(T)\right\}\right) \geq 0 .
$$

In view of the properties of $\bar{\phi}$, we deduce from this inequality that

$$
\sum_{i=1}^{p} u_{i}\left[F_{i}(S)-\lambda G_{i}(S)\right]+\sum_{j=1}^{q} v_{j} H_{j}(S)-\left\{\sum_{i=1}^{p} u_{i}\left[F_{i}(T)-\lambda G_{i}(T)\right]+\sum_{j=1}^{q} v_{j} H_{j}(T)\right\} \geq 0,
$$

which, because of (3.3), (3.4), primal feasibility of $S$, and nonnegativity of $v$, reduces to (3.16), and so the rest of the proof is identical to that of part (a).

Theorem 3.2 (strong duality). Let $S^{*}$ be a regular optimal solution of $(P)$, let $\mathscr{F}\left(S, S^{*}\right.$; $\left.D F\left(S^{*}\right)\right)=\sum_{k=1}^{n}\left\langle D_{k} F\left(S^{*}\right), \chi_{S_{k}}-\chi_{S_{k}^{*}}\right\rangle$ for any differentiable function $F: \mathbb{A}^{n} \rightarrow \mathbb{R}$ and $S \in$ $\mathbb{A}^{n}$, and assume that any of the three sets of hypotheses specified in Theorem 3.1 holds for all feasible solutions of (DI). Then there exist $u^{*} \in U, v^{*} \in \mathbb{R}_{+}^{q}$, and $\lambda^{*} \in \mathbb{R}_{+}$such that $\left(S^{*}, u^{*}, v^{*}, \lambda^{*}\right)$ is an optimal solution of $(D I)$ and $\varphi\left(S^{*}\right)=\lambda^{*}$.

Proof. By Theorem 2.9, there exist $u^{*}, v^{*}$, and $\lambda^{*}\left(=\varphi\left(S^{*}\right)\right)$, as specified above, such that $\left(S^{*}, u^{*}, v^{*}, \lambda^{*}\right)$ is a feasible solution of (DI). Since $\varphi\left(S^{*}\right)=\lambda^{*}$, optimality of $\left(S^{*}, u^{*}, v^{*}\right.$, $\lambda^{*}$ ) for (DI) follows from Theorem 3.1.

We also have the following converse duality result for (P)-(DI).

Theorem 3.3 (strict converse duality). Let $S^{*}$ and $\mathscr{F}\left(S, S^{*} ;{ }^{*}\right)$ be as in Theorem 3.2, let $(\tilde{S}, \tilde{u}, \tilde{v}, \tilde{\lambda})$ be an optimal solution of $(D I)$, and assume that any of the following three sets of hypotheses is satisfied:

(a) the assumptions specified in Theorem 3.1(a) are satisfied for all feasible solutions of $(D I) ; F_{i}$ is strictly $\left(\mathscr{F}, b, \bar{\phi}, \bar{\rho}_{i}, \theta\right)$-univex at $\tilde{S}$ for at least one index $i \in \underline{p}$ with the corresponding component $\tilde{u}_{i}$ of $\tilde{u}$ positive, and $\bar{\phi}(a)>0 \Rightarrow a>0$, or $-G_{i}$ is strictly $\left(\mathscr{F}, b, \bar{\phi}, \hat{\rho}_{i}, \theta\right)$-univex at $\tilde{S}$ for at least one index $i \in p$ with $\tilde{u}_{i}($ and $\tilde{\lambda})$ positive, and $\bar{\phi}(a)>0 \Rightarrow a>0$, or $H_{j}$ is strictly $\left(\mathscr{F}, b, \tilde{\phi}, \tilde{\rho}_{j}, \theta\right)$-univex at $\tilde{S}$ for at least one index $j \in \underline{q}$ with $\tilde{v}_{j}$ positive, and $\tilde{\phi}(a)>0 \Rightarrow a>0$, or $\sum_{i=1}^{p} \tilde{u}_{i}\left(\bar{\rho}_{i}+\tilde{\lambda} \hat{\rho}_{i}\right)+\sum_{j=1}^{q} \tilde{v}_{j} \tilde{\rho}_{j}>0$;

(b) the assumptions specified in Theorem 3.1(b) are satisfied for all feasible solutions of (DI), $F_{i}$ and $\bar{\phi}$ or $-G_{i}$ and $\bar{\phi}$ satisfy the requirements described in part (a), or the function $R \rightarrow \sum_{j=1}^{q} \tilde{v}_{j} H_{j}(R)$ is strictly $\left(\mathscr{F}_{F}, b, \tilde{\phi}, \tilde{\rho}, \theta\right)$-pseudounivex at $\tilde{S}$, or $\sum_{i=1}^{p} \tilde{u}_{i}\left(\bar{\rho}_{i}+\tilde{\lambda} \hat{\rho}_{i}\right)+\tilde{\rho}>0$;

(c) the assumptions specified in Theorem 3.1(c) are satisfied for all feasible solutions of $(D I)$, and the function $R \rightarrow \sum_{i=1}^{p} \tilde{u}_{i}\left[F_{i}(R)-\tilde{\lambda} G_{i}(R)\right]+\sum_{j=1}^{q} \tilde{v}_{j} H_{j}(R)$ is strictly $(\mathscr{F}, b, \bar{\phi}, 0, \theta)$-pseudounivex at $\tilde{S}$, and $\bar{\phi}(a)>0 \Rightarrow a>0$.

Then $\tilde{S}=S^{*}$, that is, $\tilde{S}$ is an optimal solution of $(P)$, and $\varphi\left(S^{*}\right)=\tilde{\lambda}$. 
Proof. (a) Suppose to the contrary that $\tilde{S} \neq S^{*}$. From Theorem 3.2, we know that there exist $u^{*} \in U, v^{*} \in \mathbb{R}_{+}^{q}$, and $\lambda^{*} \in \mathbb{R}_{+}$such that $\left(S^{*}, u^{*}, v^{*}, \lambda^{*}\right)$ is an optimal solution of (DI) and $\varphi\left(S^{*}\right)=\lambda^{*}$. Proceeding as in the proof of Theorem 3.1 (a) (with $S$ replaced by $S^{*}$ and $(T, u, v, \lambda)$ by $\left.(\tilde{S}, \tilde{u}, \tilde{v}, \tilde{\lambda})\right)$, we arrive at the strict inequality $\varphi\left(S^{*}\right)>\tilde{\lambda}$, which contradicts the fact that $\varphi\left(S^{*}\right)=\lambda^{*}=\tilde{\lambda}$. Hence, we conclude that $\tilde{S}=S^{*}$ and $\varphi\left(S^{*}\right)=\tilde{\lambda}$.

(b)-(c) The proofs are similar to that of part (a).

\section{Duality model II}

In this section, we consider a slightly different version of (DI) that allows for a greater variety of generalized $(\mathscr{F}, b, \phi, \rho, \theta)$-univexity conditions under which duality can be established. This duality model has the form

$$
\text { Maximize } \lambda
$$

subject to

$$
\begin{gathered}
\mathscr{F}\left(S, T ; \sum_{i=1}^{p} u_{i}\left[D F_{i}(T)-\lambda D G_{i}(T)\right]+\sum_{j=1}^{q} v_{j} D H_{j}(T)\right) \geq 0 \quad \forall S \in \mathbb{A}^{n}, \\
u_{i}\left[F_{i}(T)-\lambda G_{i}(T)\right] \geq 0, \quad i \in \underline{p}, \\
v_{j} H_{j}(T) \geq 0, \quad j \in \underline{q}, \\
T \in \mathbb{A}^{n}, \quad u \in U, \quad v \in \mathbb{R}_{+}^{q}, \quad \lambda \in \mathbb{R}_{+},
\end{gathered}
$$

where $\mathscr{F}(S, T ; \cdot): L_{1}^{n}(X, \mathbb{A}, \mu) \rightarrow \mathbb{R}$ is a sublinear function. We next show that (DII) is a dual problem for $(\mathrm{P})$ by establishing weak and strong duality theorems. As demonstrated below, this can be accomplished under numerous sets of generalized $(\mathscr{F}, b, \phi, \rho, \theta)$ univexity conditions. In the statements and proofs of our duality theorems in this section, we use the functions $\mathscr{A}_{i}(\cdot, \lambda), \mathscr{A}(\cdot, u, \lambda)$, and $\mathscr{B}(\cdot, v)$, which were defined in Section 3.

Theorem 4.1 (weak duality). Let $S$ and $(T, u, v, \lambda)$ be arbitrary feasible solutions of $(P)$ and (DII), respectively, and assume that any of the following six sets of hypotheses is satisfied:

(a) (i) $\mathscr{A}(\cdot, u, \lambda)$ is $(\mathscr{F}, b, \bar{\phi}, \bar{\rho}, \theta)$-pseudounivex at $T$, and $\bar{\phi}(a) \geq 0 \Rightarrow a \geq 0$;

(ii) for each $j \in J_{+} \equiv J_{+}(v), H_{j}$ is $\left(\mathscr{F}, b, \tilde{\phi}_{j}, \tilde{\rho}_{j}, \theta\right)$-quasiunivex at $T$, $\tilde{\phi}_{j}$ is increasing, and $\tilde{\phi}_{j}(0)=0$;

(iii) $\bar{\rho}+\sum_{j \in J_{+}} v_{j} \tilde{\rho}_{j} \geq 0$;

(b) (i) $\mathscr{A}(\cdot, u, \lambda)$ is $(\mathscr{F}, b, \bar{\phi}, \bar{\rho}, \theta)$-pseudounivex at $T$, and $\bar{\phi}(a) \geq 0 \Rightarrow a \geq 0$;

(ii) $\mathscr{B}(\cdot, v)$ is $(\mathscr{F}, b, \tilde{\phi}, \tilde{\rho}, \theta)$-quasiunivex at $T, \tilde{\phi}$ is increasing, and $\tilde{\phi}(0)=0$;

(iii) $\bar{\rho}+\tilde{\rho} \geq 0$;

(c) (i) $\mathscr{A}(\cdot, u, \lambda)$ is prestrictly $(\mathscr{F}, b, \bar{\phi}, \bar{\rho}, \theta)$-quasiunivex at $T$, and $\bar{\phi}(a) \geq 0 \Rightarrow a \geq 0$;

(ii) for each $j \in J_{+}, H_{j}$ is $\left(\mathscr{F}, b, \tilde{\phi}_{j}, \tilde{\rho}_{j}, \theta\right)$-quasiunivex at $T, \tilde{\phi}_{j}$ is increasing, and $\tilde{\phi}_{j}(0)=0$;

(iii) $\bar{\rho}+\sum_{j \in J_{+}} v_{j} \tilde{\rho}_{j}>0$;

(d) (i) $\mathscr{A}(\cdot, u, \lambda)$ is prestrictly $(\mathscr{F}, b, \bar{\phi}, \bar{\rho}, \theta)$-quasiunivex at $T$, and $\bar{\phi}(a) \geq 0 \Rightarrow a \geq 0$;

(ii) $\mathscr{B}(\cdot, v)$ is $(\mathscr{F}, b, \tilde{\phi}, \tilde{\rho}, \theta)$-quasiunivex at $T, \tilde{\phi}$ is increasing, and $\tilde{\phi}(0)=0$;

(iii) $\bar{\rho}+\tilde{\rho}>0$; 
(e) (i) $\mathscr{A}(\cdot, u, \lambda)$ is prestrictly $(\mathscr{F}, b, \bar{\phi}, \bar{\rho}, \theta)$-quasiunivex at $T$, and $\bar{\phi}(a) \geq 0 \Rightarrow a \geq 0$;

(ii) for each $j \in J_{+}, H_{j}$ is strictly $\left(\mathscr{F}, b, \tilde{\phi}_{j}, \tilde{\rho}_{j}, \theta\right)$-pseudounivex at $T$, $\tilde{\phi}_{j}$ is increasing, and $\tilde{\phi}_{j}(0)=0$;

(iii) $\bar{\rho}+\sum_{j \in J_{+}} v_{j} \tilde{\rho}_{j} \geq 0$;

(f) (i) $\mathscr{A}(\cdot, u, \lambda)$ is prestrictly $(\mathscr{F}, b, \bar{\phi}, \bar{\rho}, \theta)$-quasiunivex at $T$, and $\bar{\phi}(a) \geq 0 \Rightarrow a \geq 0$;

(ii) $\mathscr{B}(\cdot, v)$ is strictly $(\mathscr{F}, b, \tilde{\phi}, \tilde{\rho}, \theta)$-pseudounivex at $T, \tilde{\phi}$ is increasing, and $\tilde{\phi}(0)=$ 0 ;

Then $\varphi(S) \geq \lambda$.

(iii) $\bar{\rho}+\tilde{\rho} \geq 0$.

Proof. (a) From the primal feasibility of $S$ and (4.3), it is clear that for each $j \in J_{+}, H_{j}(S) \leq$ $H_{j}(T)$ and so using the properties of $\tilde{\phi}_{j}$, we obtain $\tilde{\phi}_{j}\left(H_{j}(S)-H_{j}(T)\right) \leq 0$, which by virtue of (ii) implies that for each $j \in J_{+}$,

$$
\mathscr{F}\left(S, T ; b(S, T) D H_{j}(T)\right) \leq-\tilde{\rho}_{j} d^{2}(\theta(S, T)) .
$$

Since $v \geq 0, v_{j}=0$ for each $j \in q \backslash J_{+}$, and $\mathscr{F}(S, T ; \cdot)$ is sublinear, the above inequalities can be combined as follows:

$$
\mathscr{F}\left(S, T ; b(S, T) \sum_{j=1}^{q} v_{j} D H_{j}(T)\right) \leq-\sum_{j \in J_{+}} v_{j} \tilde{\rho}_{j} d^{2}(\theta(S, T)) .
$$

From (3.13) (which is valid for the present case because $b(S, T)>0, \mathscr{F}(S, T ; \cdot)$ is sublinear, and (4.1) holds) and (4.6), we see that

$$
\mathscr{F}\left(S, T ; b(S, T) \sum_{i=1}^{p} u_{i}\left[D F_{i}(T)-\lambda D G_{i}(T)\right]\right) \geq \sum_{j \in J_{+}} v_{j} \tilde{\rho}_{j} d^{2}(\theta(S, T)) \geq-\bar{\rho} d^{2}(\theta(S, T)),
$$

where the second inequality follows from (iii). In view of (i), (4.7) implies that

$$
\bar{\phi}(\mathscr{A}(S, u, \lambda)-\mathscr{A}(T, u, \lambda)) \geq 0,
$$

which in view of the properties of $\bar{\phi}$ reduces to $\mathscr{A}(S, u, \lambda)-\mathscr{A}(T, u, \lambda) \geq 0$. But $\mathscr{A}(T, u, \lambda)$ $\geq 0$ because of (4.2) and hence we have that $\mathscr{A}(S, u, \lambda) \geq 0$. This is, of course, (3.16). Therefore, the rest of the proof is identical to that of Theorem 3.1(a).

(b) The proof is similar to that of part (a).

(c) Proceeding as in the proof of part (a), we arrive at the strict inequality

$$
\mathscr{F}\left(S, T ; b(S, T) \sum_{i=1}^{p} u_{i}\left[D F_{i}(T)-\lambda D G_{i}(T)\right]\right)>-\bar{\rho} d^{2}(\theta(S, T)),
$$

which in view of (i) implies that $\bar{\phi}(\mathscr{A}(S, u, \lambda)-\mathscr{A}(T, u, \lambda)) \geq 0$. Since $\bar{\phi}(a) \geq 0 \Rightarrow a \geq 0$ and (4.2) holds, this inequality reduces to $\mathscr{A}(S, u, \lambda) \geq 0$, which leads, as seen in the proof of Theorem 3.1, to the desired conclusion that $\varphi(S) \geq \lambda$.

(d)-(f) The proofs are similar to that of part (c). 
Theorem 4.2 (weak duality). Let $S$ and $(T, u, v, \lambda)$ be arbitrary feasible solutions of $(P)$ and (DII), respectively, and assume that any of the following six sets of hypotheses is satisfied:

(a) (i) for each $i \in I_{+} \equiv I_{+}(u), \mathscr{A}_{i}(\cdot, \lambda)$ is $\left(\mathscr{F}, b, \bar{\phi}_{i}, \bar{\rho}_{i}, \theta\right)$-pseudounivex at $T, \bar{\phi}_{i}$ is increasing, and $\bar{\phi}_{i}(0)=0$;

(ii) for each $j \in J_{+} \equiv J_{+}(v), H_{j}$ is $\left(\mathscr{F}, b, \tilde{\phi}_{j}, \tilde{\rho}_{j}, \theta\right)$-quasiunivex at $T$, $\tilde{\phi}_{j}$ is increasing, and $\tilde{\phi}_{j}(0)=0$;

(iii) $\rho^{\circ}+\sum_{j \in J_{+}} v_{j} \tilde{\rho}_{j} \geq 0$, where $\rho^{\circ}=\sum_{i \in I_{+}} u_{i} \bar{\rho}_{i}$;

(b) (i) for each $i \in I_{+}, \mathscr{A}_{i}(\cdot, \lambda)$ is $\left(\mathscr{F}_{F}, b, \bar{\phi}_{i}, \bar{\rho}_{i}, \theta\right)$-pseudounivex at $T, \bar{\phi}_{i}$ is increasing, and $\bar{\phi}_{i}(0)=0$;

(ii) $\mathscr{B}(\cdot, v)$ is $(\mathscr{F}, b, \tilde{\phi}, \tilde{\rho}, \theta)$-quasiunivex at $T, \tilde{\phi}$ is increasing, and $\tilde{\phi}(0)=0$;

(iii) $\rho^{\circ}+\tilde{\rho} \geq 0$;

(c) (i) for each $i \in I_{+}, \mathscr{A}_{i}(\cdot, \lambda)$ is prestrictly $\left(\mathscr{F}, b, \bar{\phi}_{i}, \bar{\rho}_{i}, \theta\right)$-quasiunivex at $T, \bar{\phi}_{i}$ is increasing, and $\bar{\phi}_{i}(0)=0$;

(ii) for each $j \in J_{+}, H_{j}$ is $\left(\mathscr{F}, b, \tilde{\phi}_{j}, \tilde{\rho}_{j}, \theta\right)$-quasiunivex at $T, \tilde{\phi}_{j}$ is increasing, and $\tilde{\phi}_{j}(0)=0$;

(iii) $\rho^{\circ}+\sum_{j \in J_{+}} v_{j} \tilde{\rho}_{j}>0$;

(d) (i) for each $i \in I_{+}, \mathscr{A}_{i}(\cdot, \lambda)$ is prestrictly $\left(\mathscr{F}, b, \bar{\phi}_{i}, \bar{\rho}_{i}, \theta\right)$-quasiunivex at $T, \bar{\phi}_{i}$ is increasing, and $\bar{\phi}_{i}(0)=0$;

(ii) $\mathscr{B}(\cdot, v)$ is $(\mathscr{F}, b, \tilde{\phi}, \tilde{\rho}, \theta)$-quasiunivex at $T, \tilde{\phi}$ is increasing, and $\tilde{\phi}(0)=0$;

(iii) $\rho^{\circ}+\tilde{\rho}>0$;

(e) (i) for each $i \in I_{+}, \mathscr{A}_{i}(\cdot, \lambda)$ is prestrictly $\left(\mathscr{F}, b, \bar{\phi}_{i}, \bar{\rho}_{i}, \theta\right)$-quasiunivex at $T, \bar{\phi}_{i}$ is increasing, and $\bar{\phi}_{i}(0)=0$;

(ii) for each $j \in J_{+}, H_{j}$ is strictly $\left(\mathscr{F}_{F}, b, \tilde{\phi}_{j}, \tilde{\rho}_{j}, \theta\right)$-pseudounivex at $T$, $\tilde{\phi}_{j}$ is increasing, and $\tilde{\phi}_{j}(0)=0$;

(iii) $\rho^{\circ}+\sum_{j \in J_{+}} v_{j} \tilde{\rho}_{j} \geq 0$;

(f) (i) for each $i \in I_{+}, \mathscr{A}_{i}(\cdot, \lambda)$ is prestrictly $\left(\mathscr{F}, b, \bar{\phi}_{i}, \bar{\rho}_{i}, \theta\right)$-quasiunivex at $T, \bar{\phi}_{i}$ is increasing, and $\bar{\phi}_{i}(0)=0$;

(ii) $\mathscr{B}(\cdot, v)$ is strictly $(\mathscr{F}, b, \tilde{\phi}, \tilde{\rho}, \theta)$-pseudounivex at $T, \tilde{\phi}$ is increasing, and $\tilde{\phi}(0)=$ 0 ;

Then $\varphi(S) \geq \lambda$.

(iii) $\rho^{\circ}+\tilde{\rho} \geq 0$.

Proof. (a) Suppose to the contrary that $\varphi(S)<\lambda$. This implies that for each $i \in p, F_{i}(S)-$ $\lambda G_{i}(S)<0$. From this and (4.2), we deduce that for each $i \in I_{+}$,

$$
F_{i}(S)-\lambda G_{i}(S)<0 \leq F_{i}(T)-\lambda G_{i}(T),
$$

which in view of the properties of $\bar{\phi}_{i}$ can be expressed as follows:

$$
\bar{\phi}_{i}\left(\mathscr{A}_{i}(S, \lambda)-\mathscr{A}_{i}(T, \lambda)\right)<0 .
$$

By virtue of (i), these inequalities imply that for each $i \in I_{+}$,

$$
\mathscr{F}_{F}\left(S, T ; b(S, T)\left[D F_{i}(T)-\lambda D G_{i}(T)\right]\right)<-\bar{\rho}_{i} d^{2}(\theta(S, T)) .
$$




\section{Minmax fractional subset programming}

Inasmuch as $u \geq 0, u_{i}=0$ for each $i \in \underline{p} \backslash I_{+}, \sum_{i \in I_{+}} u_{i}=1$, and $\mathscr{F}(S, T ; \cdot)$ is sublinear, the above inequalities yield

$$
\mathscr{F}\left(S, T ; b(S, T) \sum_{i=1}^{p} u_{i}\left[D F_{i}(T)-\lambda D G_{i}(T)\right]\right)<-\sum_{i \in I_{+}} u_{i} \bar{\rho}_{i} d^{2}(\theta(S, T)) .
$$

From (3.13) (which is valid for the present case because $b(S, T)>0, \mathscr{F}(S, T ; \cdot)$ is sublinear, and (4.1) holds), (4.13), and (iii), we infer that

$$
\mathscr{F}\left(S, T ; b(S, T) \sum_{j=1}^{q} v_{j} D H_{j}(T)\right)>\rho^{\circ} d^{2}(\theta(S, T)) \geq-\sum_{j \in J_{+}} v_{j} \tilde{\rho}_{j} d^{2}(\theta(S, T)) .
$$

But this contradicts (4.6), which is valid for the present case because of our hypotheses set forth in (ii). Hence, $\varphi(S) \geq \lambda$.

(b) The proof is similar to that of part (a).

(c) Suppose to the contrary that $\varphi(S)<\lambda$. As seen in the proof of part (a), this supposition leads to the inequalities

$$
\bar{\phi}_{i}\left(\mathscr{A}_{i}(S, \lambda)-\mathscr{A}_{i}(T, \lambda)\right)<0, \quad i \in I_{+},
$$

which by (i) imply that for each $i \in I_{+}$,

$$
\mathscr{F}\left(S, T ; b(S, T)\left[D F_{i}(T)-\lambda D G_{i}(T)\right]\right) \leq-\bar{\rho}_{i} d^{2}(\theta(S, T)) .
$$

Now proceeding as in the proof of Theorem 4.1 and using these inequalities and (iii), we obtain

$$
\mathscr{F}\left(S, T ; b(S, T) \sum_{j=1}^{q} v_{j} D H_{j}(T)\right)>-\sum_{j \in J_{+}} v_{j} \tilde{\rho}_{j} d^{2}(\theta(S, T)),
$$

in contradiction to (4.6), which is valid for the present case because of the assumptions made in (ii). Therefore, $\varphi(S) \geq \lambda$.

(d)-(f) The proofs are similar to that of part (c).

The following theorem may be viewed as a variant of Theorem 4.2; its proof is almost identical to that of Theorem 4.2 and hence omitted.

Theorem 4.3 (weak duality). Let $S$ and $(T, u, v, \lambda)$ be arbitrary feasible solutions of $(P)$ and (DII), respectively, and assume that any of the following four sets of hypotheses is satisfied:

(a) (i) for each $i \in I_{1+} \neq \varnothing, \mathscr{A}_{i}(\cdot, \lambda)$ is $\left(\mathscr{F}, b, \bar{\phi}_{i}, \bar{\rho}_{i}, \theta\right)$-pseudounivex at $T$, for each $i \in I_{2+}, \mathscr{A}_{i}(\cdot, \lambda)$ is prestrictly $\left(\mathscr{F}, b, \bar{\phi}_{i}, \bar{\rho}_{i}, \theta\right)$-quasiunivex at $T$, and for each $i \in I_{+} \equiv I_{+}(u), \bar{\phi}_{i}$ is increasing and $\bar{\phi}_{i}(0)=0$, where $\left\{I_{1+}, I_{2+}\right\}$ is a partition of $I_{+}$;

(ii) for each $j \in J_{+} \equiv J_{+}(v), H_{j}$ is $\left(\mathscr{F}_{F}, b, \tilde{\phi}_{j}, \tilde{\rho}_{j}, \theta\right)$-quasiunivex at T, $\tilde{\phi}_{j}$ is increasing, and $\tilde{\phi}_{j}(0)=0$;

(iii) $\rho^{\circ}+\sum_{j \in J_{+}} v_{j} \tilde{\rho}_{j} \geq 0$, where $\rho^{\circ}=\sum_{i \in I_{+}} u_{i} \bar{\rho}_{i}$; 
(b) (i) for each $i \in I_{1+} \neq \varnothing, \mathscr{A}_{i}(\cdot, \lambda)$ is $\left(\mathscr{F}, b, \bar{\phi}_{i}, \bar{\rho}_{i}, \theta\right)$-pseudounivex at $T$, for each $i \in I_{2+}, \mathscr{A}_{i}(\cdot, \lambda)$ is prestrictly $\left(\mathscr{F}, b, \bar{\phi}_{i}, \bar{\rho}_{i}, \theta\right)$-quasiunivex at $T$, and for each $i \in I_{+}, \bar{\phi}_{i}$ is increasing and $\bar{\phi}_{i}(0)=0$, where $\left\{I_{1+}, I_{2+}\right\}$ is a partition of $I_{+}$;

(ii) $\mathscr{B}(\cdot, v)$ is $(\mathscr{F}, b, \tilde{\phi}, \tilde{\rho}, \theta)$-quasiunivex at $T, \tilde{\phi}$ is increasing, and $\tilde{\phi}(0)=0$;

(iii) $\rho^{\circ}+\tilde{\rho} \geq 0$;

(c) (i) for each $i \in I_{+}, \mathscr{A}_{i}(\cdot, \lambda)$ is prestrictly $\left(\mathscr{F}, b, \bar{\phi}_{i}, \bar{\rho}_{i}, \theta\right)$-quasiunivex at $T, \bar{\phi}_{i}$ is increasing, and $\bar{\phi}_{i}(0)=0$;

(ii) for each $j \in J_{1+}, H_{j}$ is $\left(\mathscr{F}_{F}, b, \tilde{\phi}_{j}, \tilde{\rho}_{j}, \theta\right)$-quasiunivex at $T$, for each $j \in J_{2+} \neq$ $\varnothing, H_{j}$ is strictly $\left(\mathscr{F}_{F}, b, \tilde{\phi}_{j}, \tilde{\rho}_{j}, \theta\right)$-pseudounivex at $T$, and for each $j \in J_{+}, \tilde{\phi}_{j}$ is increasing and $\tilde{\phi}_{j}(0)=0$, where $\left\{J_{1+}, J_{2+}\right\}$ is a partition of $J_{+}$;

(iii) $\rho^{\circ}+\sum_{j \in J_{+}} v_{j} \tilde{\rho}_{j} \geq 0$;

(d) (i) for each $i \in I_{1+}, \mathscr{A}_{i}(\cdot, \lambda)$ is $\left(\mathscr{F}, b, \bar{\phi}_{i}, \bar{\rho}_{i}, \theta\right)$-pseudounivex at $T$, for each $i \in I_{2+}$, $\mathscr{A}_{i}(\cdot, \lambda)$ is prestrictly $\left(\mathscr{F}, b, \bar{\phi}_{i}, \bar{\rho}_{i}, \theta\right)$-quasiunivex at $T$, and for each $i \in I_{+}, \bar{\phi}_{i}$ is increasing and $\bar{\phi}_{i}(0)=0$, where $\left\{I_{1+}, I_{2+}\right\}$ is a partition of $I_{+}$;

(ii) for each $j \in J_{1+}, H_{j}$ is $\left(\mathscr{F}, b, \tilde{\phi}_{j}, \tilde{\rho}_{j}, \theta\right)$-quasiunivex at $T$, for each $j \in J_{2+}, H_{j}$ is strictly $\left(\mathscr{F}, b, \tilde{\phi}_{j}, \tilde{\rho}_{j}, \theta\right)$-pseudounivex at $T$, and for each $j \in J_{+}, \tilde{\phi}_{j}$ is increasing and $\tilde{\phi}_{j}(0)=0$, where $\left\{J_{1+}, J_{2+}\right\}$ is a partition of $J_{+}$;

Then $\varphi(S) \geq \lambda$.

(iii) $\rho^{\circ}+\sum_{j \in J_{+}} v_{j} \tilde{\rho}_{j} \geq 0$;

(iv) $I_{1+} \neq \varnothing, J_{2+} \neq \varnothing$, or $\rho^{\circ}+\sum_{j \in J_{+}} v_{j} \tilde{\rho}_{j}>0$.

Theorem 4.4 (strong duality). Let $S^{*}$ be a regular optimal solution of $(P)$, let $\mathscr{F}\left(S, S^{*}\right.$; $\left.D F\left(S^{*}\right)\right)=\sum_{k=1}^{n}\left\langle D_{k} F\left(S^{*}\right), \chi_{S_{k}}-\chi_{S_{k}^{*}}\right\rangle$ for any differentiable function $F: \mathbb{A}^{n} \rightarrow \mathbb{R}$ and $S \in$ $\mathbb{A}^{n}$, and assume that any of the fifteen sets of hypotheses specified in Theorems 4.1-4.3 holds for all feasible solutions of (DII). Then there exist $u^{*} \in U, v^{*} \in \mathbb{R}_{+}^{q}$, and $\lambda^{*} \in \mathbb{R}_{+}$such that $\left(S^{*}, u^{*}, v^{*}, \lambda^{*}\right)$ is an optimal solution of $(D I I)$ and $\phi\left(S^{*}\right)=\lambda^{*}$.

Proof. By Theorem 2.9, there exist $u^{*}, v^{*}$, and $\lambda^{*}$, as specified above, such that $\left(S^{*}, u^{*}, v^{*}\right.$, $\left.\lambda^{*}\right)$ is a feasible solution of (DII) and $\phi\left(S^{*}\right)=\lambda^{*}$. That $\left(S^{*}, u^{*}, v^{*}, \lambda^{*}\right)$ is optimal for (DII) follows from (the corresponding part of) Theorems 4.1-4.3.

Theorem 4.5 (strict converse duality). Let $S^{*}$ and $\mathscr{F}\left(S, S^{*} ; \cdot\right)$ be as in Theorem 4.4, let $(\tilde{S}, \tilde{u}, \tilde{v}, \tilde{\lambda})$ be an optimal solution of (DII), and assume that either one of the two sets of hypotheses specified in Theorem 4.1(a) and (b) is satisfied for all feasible solutions of (DII). Assume, furthermore, that $\mathscr{A}(\cdot, \tilde{u}, \tilde{\lambda})$ is strictly $(\mathscr{F}, b, \bar{\phi}, \bar{\rho}, \theta)$-pseudounivex at $\tilde{S}$ and that $\bar{\phi}(a)>0 \Rightarrow a>0$. Then $\tilde{S}=S^{*}$, that is, $\tilde{S}$ is an optimal solution of $(P)$, and $\varphi\left(S^{*}\right)=\tilde{\lambda}$.

Proof. Suppose to the contrary that $\tilde{S} \neq S^{*}$. From Theorem 4.4, we know that there exist $u^{*} \in U, v^{*} \in \mathbb{R}_{+}^{q}$, and $\lambda^{*} \in \mathbb{R}_{+}$such that $\left(S^{*}, u^{*}, v^{*}, \lambda^{*}\right)$ is an optimal solution of (DII) and $\varphi\left(S^{*}\right)=\lambda^{*}$. Proceeding as in the proof of Theorem 4.1(a) (with $S$ replaced by $S^{*}$ and $(T, u, v, \lambda)$ by $(\tilde{S}, \tilde{u}, \tilde{v}, \tilde{\lambda}))$, we arrive at the strict inequality $\varphi\left(S^{*}\right)>\tilde{\lambda}$, which contradicts the fact that $\varphi\left(S^{*}\right)=\lambda^{*}=\tilde{\lambda}$. Hence, we conclude that $\tilde{S}=S^{*}$ and $\varphi\left(S^{*}\right)=\tilde{\lambda}$.

Theorem 4.6 (strict converse duality). Let $S^{*}, \mathscr{F}\left(S, S^{*} ; \cdot\right)$, and $(\tilde{S}, \tilde{u}, \tilde{v}, \tilde{\lambda})$ be as in Theorem 4.5, and assume that any of the four sets of hypotheses specified in parts $(c)-(f)$ of Theorem 4.1 is satisfied for all feasible solutions of (DII). Assume, furthermore, that $\mathbb{A}(\cdot, \tilde{u}, \tilde{\lambda})$ 


\section{Minmax fractional subset programming}

is $(\mathscr{F}, b, \bar{\phi}, \bar{\rho}, \theta)$-quasiunivex at $\tilde{S}$ and that $\bar{\phi}(a)>0 \Rightarrow a>0$. Then $\tilde{S}=S^{*}$, that is, $\tilde{S}$ is an optimal solution of $(P)$, and $\varphi\left(S^{*}\right)=\tilde{\lambda}$.

Proof. The proof is similar to that of Theorem 4.5.

\section{Duality model III}

In this section, we formulate and discuss a more general duality model for $(\mathrm{P})$ with the help of a partitioning scheme that was originally proposed in [2] for constructing generalized dual problems for nonlinear programs with point functions. The flexible structure of this duality model will allow us to establish duality under various generalized $(\mathscr{F}, b, \phi, \rho, \theta)$-univexity hypotheses that can be imposed on certain combinations of the problem functions.

Let $\left\{J_{0}, J_{1}, \ldots, J_{m}\right\}$ be a partition of the index set $\underline{q}$; thus $J_{r} \subset \underline{q}$ for each $r \in\{0,1, \ldots, m\}$, $J_{r} \cap J_{s}=\varnothing$ for each $r, s \in\{0,1, \ldots, m\}$ with $r \neq s$, and $\bigcup_{r=0}^{m} J_{r}=q$. In addition, we will make use of the functions $\mathbf{A}_{i}(\cdot, v, \lambda), \mathbf{A}(\cdot, u, v, \lambda)$, and $\mathbf{B}_{t}(\cdot, v): \mathbb{A}^{n} \rightarrow \mathbb{R}$ defined, for fixed $\lambda, u$, and $v$, by

$$
\begin{gathered}
\mathbf{A}_{i}(T, v, \lambda)=F_{i}(T)-\lambda G_{i}(T)+\sum_{j \in J_{0}} v_{j} H_{j}(T), \quad i \in \underline{p}, \\
\mathbf{A}(T, u, v, \lambda)=\sum_{i=1}^{p} u_{i}\left[F_{i}(T)-\lambda G_{i}(T)\right]+\sum_{j \in J_{0}} v_{j} H_{j}(T), \\
\mathbf{B}_{t}(T, v)=\sum_{j \in J_{t}} v_{j} H_{j}(T), \quad t \in \underline{m} .
\end{gathered}
$$

Consider the following problem:

$$
\text { Maximize } \lambda
$$

subject to

$$
\begin{gathered}
\mathscr{F}\left(S, T ; \sum_{i=1}^{p} u_{i}\left[D F_{i}(T)-\lambda D G_{i}(T)\right]+\sum_{j=1}^{q} v_{j} D H_{j}(T)\right) \geq 0 \quad \forall S \in \mathbb{A}^{n}, \\
F_{i}(T)-\lambda G_{i}(T)+\sum_{j \in J_{0}} v_{j} H_{j}(T) \geq 0, \quad i \in \underline{p}, \\
\sum_{j \in J_{t}} v_{j} H_{j}(T) \geq 0, \quad t \in \underline{m}, \\
T \in \mathbb{A}^{n}, \quad u \in U, \quad v \in \mathbb{R}_{+}^{q}, \quad \lambda \in \mathbb{R}_{+},
\end{gathered}
$$

where $\mathscr{F}(S, T ; \cdot): L_{1}^{n}(X, \mathbb{A}, \mu) \rightarrow \mathbb{R}$ is a sublinear function.

We next show that (DIII) is a dual problem for $(\mathrm{P})$ by proving weak and strong duality theorems. 
THeorem 5.1 (weak duality). Let $S$ and $(T, u, v, \lambda)$ be arbitrary feasible solutions of $(P)$ and (DIII), respectively, and assume that any of the following four sets of hypotheses is satisfied:

(a) (i) $\mathbf{A}(\cdot, u, v, \lambda)$ is $(\mathscr{F}, b, \bar{\phi}, \bar{\rho}, \theta)$-pseudounivex at $T$, and $\bar{\phi}(a) \geq 0 \Rightarrow a \geq 0$;

(ii) for each $t \in \underline{m}, \mathbf{B}_{t}(\cdot, v)$ is $\left(\mathscr{F}, b, \tilde{\phi}_{t}, \tilde{\rho}_{t}, \theta\right)$-quasiunivex at $T$, $\tilde{\phi}_{t}$ is increasing, and $\tilde{\phi}_{t}(0)=0$

(iii) $\bar{\rho}+\sum_{t=1}^{m} \tilde{\rho}_{t} \geq 0$;

(b) (i) $\mathbf{A}(\cdot, u, v, \lambda)$ is prestrictly $(\mathscr{F}, b, \bar{\phi}, \bar{\rho}, \theta)$-quasiunivex at $T$, and $\bar{\phi}(a) \geq 0 \Rightarrow a \geq$ 0 ;

(ii) for each $t \in \underline{m}, \mathbf{B}_{t}(\cdot, v)$ is strictly $\left(\mathscr{F}, b, \tilde{\phi}_{t}, \tilde{\rho}_{t}, \theta\right)$-pseudounivex at $T, \tilde{\phi}_{t}$ is increasing, and $\widetilde{\phi}_{t}(0)=0$;

(iii) $\bar{\rho}+\sum_{t=1}^{m} \tilde{\rho}_{t} \geq 0$;

(c) (i) $\mathbf{A}(\cdot, u, v, \lambda)$ is prestrictly $(\mathscr{F}, b, \bar{\phi}, \bar{\rho}, \theta)$-quasiunivex at $T$, and $\bar{\phi}(a) \geq 0 \Rightarrow a \geq$ 0 ;

(ii) for each $t \in \underline{m}, \mathbf{B}_{t}(\cdot, v)$ is $\left(\mathscr{F}, b, \tilde{\phi}_{t}, \tilde{\rho}_{t}, \theta\right)$-quasiunivex at $T$, $\tilde{\phi}_{t}$ is increasing, and $\tilde{\phi}_{t}(0)=0$

(iii) $\bar{\rho}+\sum_{t=1}^{m} \tilde{\rho}_{t}>0$;

(d) (i) $\mathbf{A}(\cdot, u, v, \lambda)$ is prestrictly $(\mathscr{F}, b, \bar{\phi}, \bar{\rho}, \theta)$-quasiunivex at $T$, and $\bar{\phi}(a) \geq 0 \Rightarrow a \geq$ 0 ;

(ii) for each $t \in \underline{m_{1}}, \mathbf{B}_{t}(\cdot, v)$ is $\left(\mathscr{F}, b, \tilde{\phi}_{t}, \tilde{\rho}_{t}, \theta\right)$-quasiunivex at $T$, $\tilde{\phi}_{t}$ is increasing, and $\tilde{\phi}_{t}(0)=0$, for each $t \in \underline{m_{2}} \neq \varnothing, \mathbf{B}_{t}(\cdot, v)$ is strictly $\left(\mathscr{F}_{t}, b, \tilde{\phi}_{t}, \tilde{\rho}_{t}, \theta\right)$ pseudounivex at T, $\tilde{\phi}_{t}$ is increasing, and $\tilde{\phi}_{t}(0)=0$, where $\left\{\underline{m_{1}}, \underline{m_{2}}\right\}$ is a partition of $\underline{m}$;

Then $\varphi(S) \geq \lambda$.

(iii) $\bar{\rho}+\sum_{t=1}^{m} \tilde{\rho}_{t} \geq 0$.

Proof. (a) From the nonnegativity of $b(S, T)$, sublinearity of $\mathscr{F}(S, T ; \cdot)$, and (5.2), it follows that

$$
\begin{gathered}
\mathscr{F}\left(S, T ; b(S, T)\left\{\sum_{i=1}^{p} u_{i}\left[D F_{i}(T)-\lambda D G_{i}(T)\right]+\sum_{j \in J_{0}} v_{j} D H_{j}(T)\right\}\right) \\
+\mathscr{F}\left(S, T ; b(S, T) \sum_{t=1}^{m} \sum_{j \in J_{t}} v_{j} D H_{j}(T)\right) \geq 0 .
\end{gathered}
$$

Since $S \in \mathbb{F}$ and $v \geq 0$, it is clear from (5.4) that for each $t \in \underline{m}$,

$$
\mathbf{B}_{t}(S, v)=\sum_{j \in J_{t}} v_{j} H_{j}(S) \leq \sum_{j \in J_{t}} v_{j} H_{j}(T)=\mathbf{B}_{t}(T, v)
$$

and so using the properties of $\tilde{\phi}_{t}$, we get

$$
\tilde{\phi}_{t}\left(\mathbf{B}_{t}(S, v)-\mathbf{B}_{t}(T, v)\right) \leq 0,
$$


1616 Minmax fractional subset programming

which in view of (ii) implies that for each $t \in \underline{m}$,

$$
\mathscr{F}\left(S, T ; b(S, T) \sum_{j \in J_{t}} v_{j} D H_{j}(T)\right) \leq-\tilde{\rho}_{t} d^{2}(\theta(S, T)) .
$$

Adding these inequalities and using the sublinearity of $\mathscr{F}(S, T ; \cdot)$, we obtain

$$
\mathscr{F}\left(S, T ; b(S, T) \sum_{t=1}^{m} \sum_{j \in J_{t}} v_{j} D H_{j}(T)\right) \leq-\sum_{t=1}^{m} \tilde{\rho}_{t} d^{2}(\theta(S, T)) .
$$

From (5.6) and (5.10), we deduce that

$$
\begin{gathered}
\mathscr{F}\left(S, T ; b(S, T)\left\{\sum_{i=1}^{p} u_{i}\left[D F_{i}(T)-\lambda D G_{i}(T)\right]+\sum_{j \in J_{0}} v_{j} D H_{j}(T)\right\}\right) \\
\geq \sum_{t=1}^{m} \tilde{\rho}_{t} d^{2}(\theta(S, T)) \geq-\bar{\rho} d^{2}(\theta(S, T)),
\end{gathered}
$$

where the second inequality follows from (iii). Because of (i), this inequality implies that

$$
\bar{\phi}(\mathbf{A}(S, u, v, \lambda)-\mathbf{A}(T, u, v, \lambda)) \geq 0 .
$$

But $\bar{\phi}(a) \geq 0 \Rightarrow a \geq 0$, and so we get $\mathbf{A}(S, u, v, \lambda)-\mathbf{A}(T, u, v, \lambda) \geq 0$, which in view of the nonnegativity of $u$ and (5.3) reduces to $\mathbf{A}(S, u, v, \lambda) \geq 0$. As $v \geq 0$ and $S \in \mathbb{F}$, we have

$$
\sum_{i=1}^{p} u_{i}\left[F_{i}(S)-\lambda G_{i}(S)\right] \geq \mathbf{A}(S, u, v, \lambda) \geq 0
$$

Now using Lemma 2.10 and this inequality, as in the proof of Theorem 3.1, we obtain the desired conclusion that $\varphi(S) \geq \lambda$.

(b) - (d) The proofs are similar to that of part (a).

Theorem 5.2 (weak duality). Let $S$ and $(T, u, v, \lambda)$ be arbitrary feasible solutions of $(P)$ and (DIII), respectively, and assume that any of the following six sets of hypotheses is satisfied:

(a) (i) for each $i \in I_{+} \equiv I_{+}(u), \mathbf{A}_{i}(\cdot, v, \lambda)$ is $\left(\mathscr{F}, b, \bar{\phi}_{i}, \bar{\rho}_{i}, \theta\right)$-pseudounivex at $T, \bar{\phi}_{i}$ is increasing, and $\bar{\phi}_{i}(0)=0$;

(ii) for each $t \in \underline{m}, \mathbf{B}_{t}(\cdot, v)$ is $\left(\mathscr{F}, b, \tilde{\phi}_{t}, \tilde{\rho}_{t}, \theta\right)$-quasiunivex at $T$, $\tilde{\phi}_{t}$ is increasing, and $\tilde{\phi}_{t}(0)=0$

(iii) $\sum_{i \in I_{+}} u_{i} \bar{\rho}_{i}+\sum_{t=1}^{m} \tilde{\rho}_{t} \geq 0$;

(b) (i) for each $i \in I_{+}, \mathbf{A}_{i}(\cdot, v, \lambda)$ is prestrictly $\left(\mathscr{F}, b, \bar{\phi}_{i}, \bar{\rho}_{i}, \theta\right)$-quasiunivex at $T, \bar{\phi}_{i}$ is increasing, and $\bar{\phi}_{i}(0)=0$;

(ii) for each $t \in \underline{m}, \mathbf{B}_{t}(\cdot, v)$ is strictly $\left(\mathscr{F}, b, \tilde{\phi}_{t}, \tilde{\rho}_{t}, \theta\right)$-pseudounivex at $T, \tilde{\phi}_{t}$ is increasing, and $\widetilde{\phi}_{t}(0)=0$;

(iii) $\sum_{i \in I_{+}} u_{i} \bar{\rho}_{i}+\sum_{t=1}^{m} \tilde{\rho}_{t} \geq 0$; 
(c) (i) for each $i \in I_{+}, \mathbf{A}_{i}(\cdot, v, \lambda)$ is prestrictly $\left(\mathscr{F}, b, \bar{\phi}_{i}, \bar{\rho}_{i}, \theta\right)$-quasiunivex at $T, \bar{\phi}_{i}$ is increasing, and $\bar{\phi}_{i}(0)=0$;

(ii) for each $t \in \underline{m}, \mathbf{B}_{t}(\cdot, v)$ is $\left(\mathscr{F}_{F}, b, \tilde{\phi}_{t}, \tilde{\rho}_{t}, \theta\right)$-quasiunivex at $T$, $\tilde{\phi}_{t}$ is increasing, and $\tilde{\phi}_{t}(0)=0$;

(iii) $\sum_{i \in I_{+}} u_{i} \bar{\rho}_{i}+\sum_{t=1}^{m} \tilde{\rho}_{t}>0$;

(d) (i) for each $i \in I_{1+} \neq \varnothing, \mathbf{A}_{i}(\cdot, v, \lambda)$ is $\left(\mathscr{F}, b, \bar{\phi}_{i}, \bar{\rho}_{i}, \theta\right)$-pseudounivex at $T$, for each $i \in I_{2+}, \mathbf{A}_{i}(\cdot, v, \lambda)$ is prestrictly $\left(\mathscr{F}, b, \bar{\phi}_{i}, \bar{\rho}_{i}, \theta\right)$-quasiunivex at $T$, for each $i \in I_{+}$, $\bar{\phi}_{i}$ is increasing, and $\bar{\phi}_{i}(0)=0$, where $\left\{I_{1+}, I_{2+}\right\}$ is a partition of $I_{+}$;

(ii) for each $t \in \underline{m}, \mathbf{B}_{t}(\cdot, v)$ is $\left(\mathscr{F}_{F}, b, \tilde{\phi}_{t}, \tilde{\rho}_{t}, \theta\right)$-quasiunivex at $T$, $\tilde{\phi}_{t}$ is increasing, and $\tilde{\phi}_{t}(0)=0$;

(iii) $\sum_{i \in I_{+}} u_{i} \bar{\rho}_{i}+\sum_{t=1}^{m} \tilde{\rho}_{t} \geq 0$;

(e) (i) for each $i \in I_{+}, \mathbf{A}_{i}(\cdot, v, \lambda)$ is prestrictly $\left(\mathscr{F}, b, \bar{\phi}_{i}, \bar{\rho}_{i}, \theta\right)$-quasiunivex at $T, \bar{\phi}_{i}$ is increasing, and $\bar{\phi}_{i}(0)=0$;

(ii) for each $t \in m_{1} \neq \varnothing, \mathbf{B}_{t}(\cdot, v)$ is strictly $\left(\mathscr{F}, b, \tilde{\phi}_{t}, \tilde{\rho}_{t}, \theta\right)$-pseudounivex at $T, \tilde{\phi}_{t}$ is increasing, and $\tilde{\phi}_{t}(0)=0$, and for each $t \in m_{2}, \mathbf{B}_{t}(\cdot, v)$ is $\left(\mathscr{F}_{F}, b, \tilde{\phi}_{t}, \tilde{\rho}_{t}, \theta\right)$ quasiunivex at $T, \tilde{\phi}_{t}$ is increasing, and $\tilde{\phi}_{t}(0)=0$, where $\left\{m_{1}, m_{2}\right\}$ is a partition of $\underline{m}$;

(iii) $\sum_{i \in I_{+}} u_{i} \bar{\rho}_{i}+\sum_{t=1}^{m} \tilde{\rho}_{t} \geq 0$;

(f) (i) for each $i \in I_{1+}, \mathbf{A}_{i}(\cdot, v, \lambda)$ is $\left(\mathscr{F}, b, \bar{\phi}_{i}, \bar{\rho}_{i}, \theta\right)$-pseudounivex at $T, \bar{\phi}_{i}$ is increasing, and $\bar{\phi}_{i}(0)=0$, and for each $i \in I_{2+}, \mathbf{A}_{i}(\cdot, v, \lambda)$ is prestrictly $\left(\mathscr{F}, b, \bar{\phi}_{i}, \bar{\rho}_{i}, \theta\right)$ quasiunivex at $T, \bar{\phi}_{i}$ is increasing, and $\bar{\phi}_{i}(0)=0$, where $\left\{I_{1+}, I_{2+}\right\}$ is a partition of $I_{+}$;

(ii) for each $t \in m_{1}, \mathbf{B}_{t}(\cdot, v)$ is strictly $\left(\mathscr{F}, b, \tilde{\phi}_{t}, \tilde{\rho}_{t}, \theta\right)$-pseudounivex at $T, \tilde{\phi}_{t}$ is increasing, and $\tilde{\phi}_{t}(0)=0$, and for each $t \in \underline{m_{2}}, \mathbf{B}_{t}(\cdot, v)$ is $\left(\mathscr{F}_{t}, b, \tilde{\phi}_{t}, \tilde{\rho}_{t}, \theta\right)$ quasiunivex at $T, \tilde{\phi}_{t}$ is increasing, and $\tilde{\phi}_{t}(0)=0$, where $\left\{m_{1}, m_{2}\right\}$ is a partition of $\underline{m}$;

(iii) $\sum_{i \in I_{+}} u_{i} \bar{\rho}_{i}+\sum_{t=1}^{m} \tilde{\rho}_{t} \geq 0$;

Then $\varphi(S) \geq \lambda$.

(iv) $I_{1+} \neq \varnothing, m_{1} \neq \varnothing$, or $\sum_{i \in I_{+}} u_{i} \bar{\rho}_{i}+\sum_{t=1}^{m} \tilde{\rho}_{t}>0$.

Proof. (a) Suppose to the contrary that

$$
\varphi(S)=\max _{1 \leq i \leq p} \frac{F_{i}(S)}{G_{i}(S)}<\lambda .
$$

This implies that for each $i \in \underline{p}$,

$$
F_{i}(S)-\lambda G_{i}(S)<0 \text {. }
$$

Since for each $i \in \underline{p}$,

$$
\begin{aligned}
\mathbf{A}_{i}(S, v, \lambda) & =F_{i}(S)-\lambda G_{i}(S)+\sum_{j \in J_{0}} v_{j} H_{j}(S) \\
& \leq F_{i}(S)-\lambda G_{i}(S) \quad(\text { by the nonnegativity of } v \text { and primal feasibility of } S) \\
& <0 \quad(\text { by }(5.15)) \\
& \leq \mathbf{A}_{i}(T, v, \lambda) \quad(\text { by }(5.3)),
\end{aligned}
$$


it follows from the properties of $\bar{\phi}_{i}$ that for each $i \in p, \bar{\phi}_{i}\left(\mathbf{A}_{i}(S, v, \lambda)-\mathbf{A}_{i}(T, v, \lambda)\right)<0$, which by (i) implies that for each $i \in p$,

$$
\mathscr{F}\left(S, T ; b(S, T)\left[D F_{i}(T)-\lambda D G_{i}(T)+\sum_{j \in J_{0}} v_{j} D H_{j}(T)\right]\right)<-\bar{\rho}_{i} d^{2}(\theta(S, T)) .
$$

Inasmuch as $u \geq 0, u_{i}=0$ for each $i \in \underline{p} \backslash I_{+}, \sum_{i \in I_{+}} u_{i}=1$, and $\mathscr{F}(S, T ; \cdot)$ is sublinear, the above inequalities yield

$$
\mathscr{F}\left(S, T ; b(S, T)\left[\sum_{i=1}^{p} u_{i}\left[D F_{i}(T)-\lambda D G_{i}(T)\right]+\sum_{j \in J_{0}} v_{j} D H_{j}(T)\right]\right)<-\sum_{i \in I_{+}} u_{i} \bar{\rho}_{i} d^{2}(\theta(S, T)) .
$$

Comparing (5.6) and (5.18), we observe that

$$
\mathscr{F}\left(S, T ; b(S, T) \sum_{t=1}^{m} \sum_{j \in J_{t}} v_{j} D H_{j}(T)\right)>\sum_{i=1}^{p} u_{i} \bar{\rho}_{i} d^{2}(\theta(S, T))>-\sum_{t=1}^{m} \tilde{\rho}_{t} d^{2}(\theta(S, T)),
$$

where the second inequality follows from (iii). Obviously, this inequality contradicts (5.10), which is valid for the present case because of (ii). Hence, we must have $\varphi(S) \geq \lambda$.

(b)-(f) The proofs are similar to that of part (a).

Theorem 5.3 (strong duality). Let $S^{*}$ be a regular optimal solution of $(P)$, let $\mathscr{F}\left(S, S^{*}\right.$; $\left.D F\left(S^{*}\right)\right)=\sum_{k=1}^{n}\left\langle D_{k} F\left(S^{*}\right), \chi_{S_{k}}-\chi_{S_{k}^{*}}\right\rangle$ for any differentiable function $F: \mathbb{A}^{n} \rightarrow \mathbb{R}$ and $S \in$ $\mathbb{A}^{n}$, and assume that any of the ten sets of hypotheses specified in Theorems 5.1 and 5.2 holds for all feasible solutions of (DIII). Then there exist $u^{*} \in U, v^{*} \in \mathbb{R}_{+}^{q}$, and $\lambda^{*} \in \mathbb{R}_{+}$such that $\left(S^{*}, u^{*}, v^{*}, \lambda^{*}\right)$ is an optimal solution of (DIII) and $\varphi\left(S^{*}\right)=\lambda^{*}$.

Proof. The proof is similar to that of Theorem 4.4.

We next show that certain modifications in Theorem 5.1 lead to a number of strict converse duality results for (P)-(DIII).

Theorem 5.4 (strict converse duality). Let $S^{*}$ and $\mathscr{F}$ be as in Theorem 5.3, let $(\tilde{S}, \tilde{u}, \tilde{v}, \tilde{\lambda})$ be an optimal solution of (DIII), and assume that any of the four sets of hypotheses specified in Theorem 5.1 is satisfied for all feasible solutions of (DIII), and that $\mathbf{A}(\cdot, \tilde{u}, \tilde{v}, \tilde{\lambda})$ is strictly $(\mathscr{F}, b, \bar{\phi}, \bar{\rho}, \theta)$-pseudounivex at $\tilde{S}$, and $\tilde{\phi}(a)>0 \Rightarrow a>0$. Then $\tilde{S}=S^{*}$ and $\varphi\left(S^{*}\right)=\tilde{\lambda}$.

Proof. We prove the theorem under the assumption that the conditions specified in Theorem 5.1(a) hold; the proofs with the conditions given in parts (b)-(d) are similar. Suppose to the contrary that $\tilde{S} \neq S^{*}$. Since $S^{*}$ is a regular optimal solution of (P), by Theorem 5.3, there exist $u^{*} \in U, v^{*} \in \mathbb{R}_{+}^{q}$, and $\lambda^{*} \in \mathbb{R}_{+}$such that $\left(S^{*}, u^{*}, v^{*}, \lambda^{*}\right)$ is an optimal solution of (DIII) and $\varphi\left(S^{*}\right)=\lambda^{*}$. Proceeding as in the proof of Theorem 5.1(a) 
(with $S$ replaced by $S^{*}$ and $(T, u, v, \lambda)$ by $(\tilde{S}, \tilde{u}, \tilde{v}, \tilde{\lambda})$ ), we arrive at the inequality

$$
\mathscr{F}\left(S^{*}, \tilde{S} ; b\left(S^{*}, \tilde{S}\right)\left\{\sum_{i=1}^{p} \tilde{u}_{i}\left[D F_{i}(\tilde{S})-\tilde{\lambda} D G_{i}(\tilde{S})\right]+\sum_{j \in J_{0}} \tilde{v}_{j} H_{j}(\tilde{S})\right\}\right) \geq-\bar{\rho} d^{2}\left(\theta\left(S^{*}, \tilde{S}\right)\right) .
$$

Since by hypothesis, $\tilde{S} \neq S^{*}$ and $\mathbf{A}(\cdot, \tilde{u}, \tilde{v}, \tilde{\lambda})$ is strictly $(\mathscr{F}, \bar{b}, \bar{\phi}, \bar{\rho}, \theta)$-pseudounivex at $\tilde{S}$, the above inequality implies that

$$
\bar{\phi}\left(\mathbf{A}\left(S^{*}, \tilde{u}, \tilde{v}, \tilde{\lambda}\right)-\mathbf{A}(\tilde{S}, \tilde{u}, \tilde{v}, \tilde{\lambda})\right)>0 .
$$

But $\bar{\phi}(a)>0 \Rightarrow a>0$, and so the above inequality yields $\mathbf{A}\left(S^{*}, \tilde{u}, \tilde{v}, \tilde{\lambda}\right)-\mathbf{A}(\tilde{S}, \tilde{u}, \tilde{v}, \tilde{\lambda})>0$, which in view of the dual feasibility of $(\tilde{S}, \tilde{u}, \tilde{v}, \tilde{\lambda})$ reduces to $\mathbf{A}\left(S^{*}, \tilde{u}, \tilde{v}, \tilde{\lambda}\right)>0$. Inasmuch as $\tilde{v} \geq 0$ and $S^{*} \in \mathbb{F}$, this inequality yields

$$
\sum_{i=1}^{p} \tilde{u}_{i}\left[F_{i}\left(S^{*}\right)-\tilde{\lambda} G_{i}\left(S^{*}\right)\right]>0
$$

Now using Lemma 2.10 and this inequality, we find that

$$
\varphi\left(S^{*}\right)=\max _{1 \leq i \leq p} \frac{F_{i}\left(S^{*}\right)}{G_{i}\left(S^{*}\right)}=\max _{u \in U} \frac{\sum_{i=1}^{p} u_{i} F_{i}\left(S^{*}\right)}{\sum_{i=1}^{p} u_{i} G_{i}\left(S^{*}\right)} \geq \frac{\sum_{i=1}^{p} \tilde{u}_{i} F_{i}\left(S^{*}\right)}{\sum_{i=1}^{p} \tilde{u}_{i} G_{i}\left(S^{*}\right)}>\tilde{\lambda},
$$

which contradicts the fact that $\varphi\left(S^{*}\right)=\lambda^{*}=\tilde{\lambda}$. Hence, we conclude that $\tilde{S}=S^{*}$ and $\varphi\left(S^{*}\right)=\tilde{\lambda}$.

We conclude this section by briefly looking at the following slightly modified version of (DIII):

$$
\text { Maximize } \lambda
$$

subject to (5.2), (5.4), (5.5), and

$$
\sum_{i=1}^{p} u_{i}\left[F_{i}(T)-\lambda G_{i}(T)\right]+\sum_{j \in J_{0}} v_{j} H_{j}(T) \geq 0
$$

An examination of the statements and proofs of Theorem 5.1, Theorem 5.3 (restricted to Theorem 5.1), and Theorem 5.4 will readily reveal the fact that these theorems remain valid for the pair (P)-(DIV).

This dual problem was originally proposed and investigated in [6], where some duality results were established under $\rho$-convexity conditions. Subsequently, these results were extended by using $(\mathscr{F}, \rho)$-convexity assumptions in [4].

Evidently, (DIII) and (DIV) contain a number of important special cases, which can easily be identified by appropriate choices of the partitioning sets $J_{0}, J_{1}, \ldots, J_{m}$ and the sublinear function $\mathscr{F}(S, T ; \cdot)$. 


\section{Minmax fractional subset programming}

\section{References}

[1] H. W. Corley, Optimization theory for n-set functions, J. Math. Anal. Appl. 127 (1987), no. 1, 193-205.

[2] B. Mond and T. Weir, Generalized Concavity and Duality, Generalized Concavity in Optimization and Economics (S. Schaible and W. T. Ziemba, eds.), Academic Press, New York, 1981, pp. 263-279.

[3] R. J. T. Morris, Optimal constrained selection of a measurable subset, J. Math. Anal. Appl. 70 (1979), no. 2, 546-562.

[4] V. Preda, On minmax programming problems containing $n$-set functions, Optimization 22 (1991), no. 4, 527-537.

[5] G. J. Zalmai, Generalized (FF, $b, \phi, \rho, \theta)$-univex functions and parameter-free duality models in minmax fractional subset programming, submitted.

[6] __ Optimality conditions and duality for constrained measurable subset selection problems with minmax objective functions, Optimization 20 (1989), no. 4, 377-395.

[7] —_ Generalized ( $\mathscr{F}, b, \phi, \rho, \theta)$-univex $n$-set functions and global parametric sufficient optimality conditions in minmax fractional subset programming, Int. J. Math. Math. Sci. 2005 (2005), no. 8, 1253-1276.

G. J. Zalmai: Department of Mathematics and Computer Science, Northern Michigan University, Marquette, MI 49855, USA

E-mail address: gzalmai@nmu.edu 


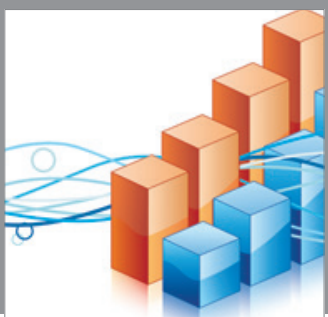

Advances in

Operations Research

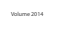

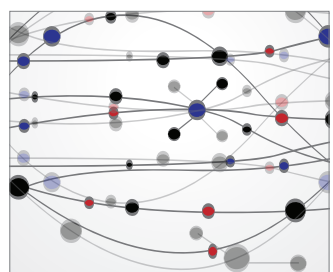

\section{The Scientific} World Journal
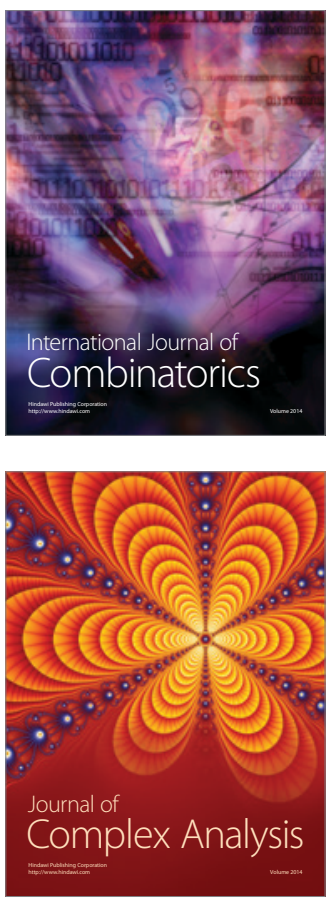

International Journal of

Mathematics and

Mathematical

Sciences
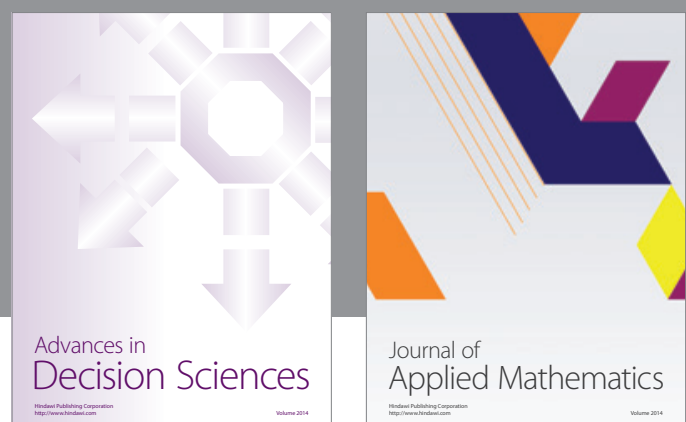

Journal of

Applied Mathematics
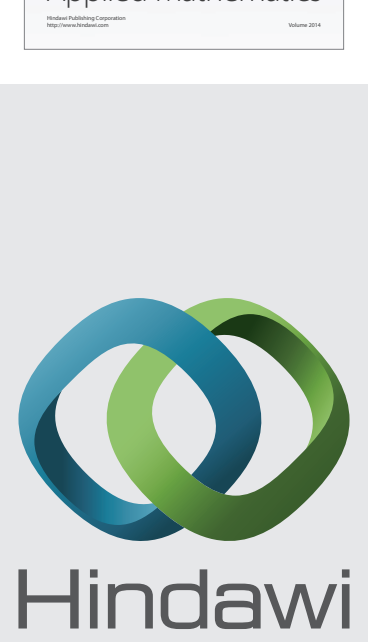

Submit your manuscripts at http://www.hindawi.com
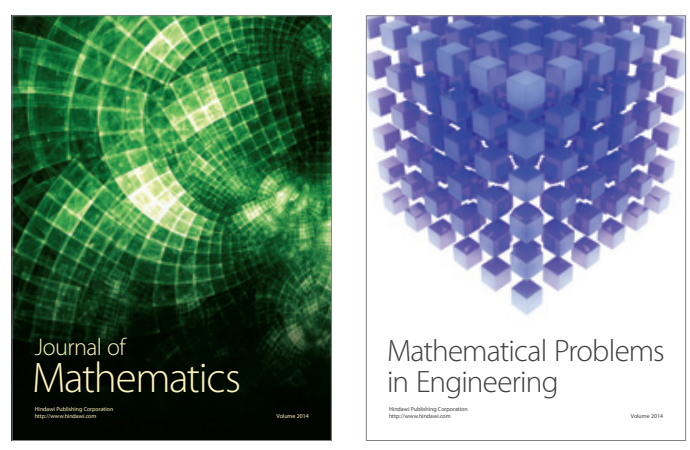

Mathematical Problems in Engineering
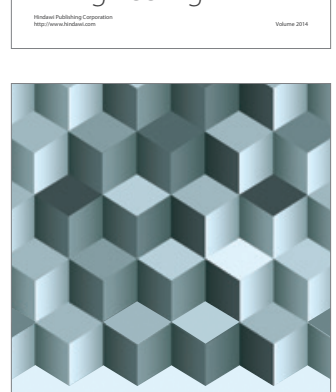

Journal of

Function Spaces
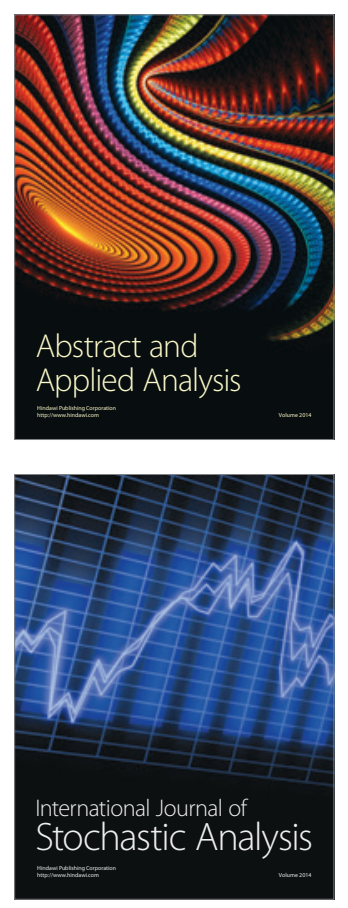

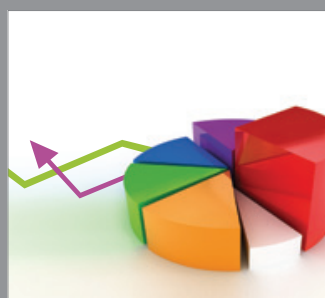

ournal of

Probability and Statistics

Promensencen
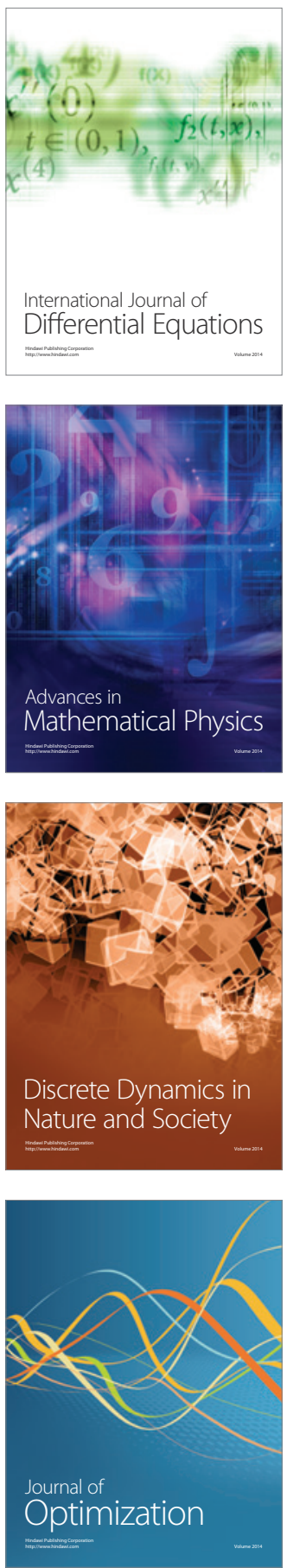